through boiling has been proved by recent experiments to be negligible. Where it is impracticable to boil instruments immediately prior to use, they may be stored in a dry state in a covered sterile receptacle instead of in spirit, or, alternatively, in a watery antiseptic solution to which a small amount of borax should be added to prevent rust. Such articles as hypodermic syringes and needles might still be stored in spirit since only \& small quantity is needed. If alcohol is used in drying the hands of surgeons it could be applied economically, but adequately, by the use of a spray after the hands have been thoroughly washed and dried with a small sterile towel. Instead of using tincture of iodine for the patient's skin before operation, the area of the operation could be painted with a watery solution of iodine or of one or other of the antiseptic dyes. For swabbing the suture line when stitches are removed after a clean operation, the application of sulphanil. amide powder can be recommended in suitable cases, or failing that an antiseptic soap may be used. For treating the backs of bedridden patients an effective method is light massage with zinc oxide and castor oil after the skin has been washed and thoroughly dried; in a small number of clinical conditions, however, the use of spirit for this purpose would be still desirable. More complete instructions will be given, no doubt, when the recommendations are issued.

\section{Electronic Engineering}

DURING the past quarter of a century, a prodigious advance has taken place in the development and application of devices utilizing free electrons, either alone or in association with positive ions, including the wide variety of radio valves, gas-filled rectifiers and cathode ray tubes, as used nowadays in communication and electrical engineering. Technical progress in this subject has hitherto been recorded as an important and ever-growing side-line in journals otherwise devoted mainly to radio communication and television, or to electrical engineering in general. As from the June issue, the Hulton Press, Ltd., has incorporated its previous monthly publication entitled Electronics, Television and Short-Wave World in a new periodical named Electronic Engineering, which is to be of such a scope as to cover the whole field of research and application of electronic devices in general.

The new journal will provide all those engaged in research and industry in this subject with a medium for interchanging ideas and learning the progress which is being made in all parts of the world. Original articles by specialists will appear regularly, and special features will include patent abstracts, reviews of books and instruments and novel circuit arrangements. An important feature, which may be expected to contribute notably to the success of the new periodical, is that Mr. G. Parr, who has been appointed editor, has been closely associated with the electrical and radio industry for more than twenty years. He is the author of a book on the cathode ray tube, and has played an important part in demonstrating the versatility of these tubes as recording and measuring instruments for laboratory and other purposes. The June issue of the journal contains the first of a series of data sheets for the use of design engineers, as well as articles on amplifiers, oscillation generators, quartz crystals and other audio- and radio-frequency problems of current interest.

\section{Cancer and Occupation in Denmark}

IN a paper on this subject read before the Danish Medical Society on November 5, 1940 (Nordisk Med., 9, 869 ; 1941), Dr. Johannes Clemmesen, of Copenhagen, maintains that cancer research has lost contact with practical medicine and that the etiology of the disease is almost exclusively studied in laboratories, while clinical mass observations are far too uncommon. The following results were obtained by him on examination of the mortality from cancer among males in various occupations in Denmark during the period 1935-1939. In agriculture and similar occupations deaths from cancer in the agegroup 45-64 were fewer than would be expected from the average of cancer deaths among the population as a whole. In industry the cancer mortality was higher than the average in accordance with the higher mortality from all causes for this group. After the sixty-fifth year the cancer mortality was the same for all occupations, but the localization varied in the different occupational groups. The cancer mortality among males and females in Denmark showed the following characteristics. In the age-groups 25-44 and 45-64 it was highest among females. In the older age-groups it was highest among males, but this excess for males was highest in Copenhagen, less in the provincial towns and not definite in the rural areas. The total cancer mortality was also highest in the capital, probably owing to the lower mortality from that cause in the agricultural than in the industrial group.

\section{Excavation of Growing Trees with Earth}

For purely horticultural purposes it is often required to remove trees and shrubs with a ball of earth for transference elsewhere, and considerable care and ingenuity must be exercised if success is to be achieved. Mr. F. A. F. Schmid and Mr. F. J. Nutman, of the East African Agricultural Research Station, Amani, Tanganyika Territory, have described (Soil Science, 49, No. 6 ; June 1940) a method devised by them during research on the water relations of coffee, for which they required large plants several years in age which could only be obtained in the field. The authors state that by their method a growing tree together with about two tons of soil has been successfully transplanted, and that only the outbreak of war has prevented further excavations. There appears, it is said, to be no limit to the size of the soil block that can be isolated in this fashion save only the size of the vehicle available for transport.

Their work was done in a district with a very light, friable, volcanic ash soil, with few stones. It is admitted that the presence of stones might make 